\title{
Recursos de la Web 2.0 aplicados en el aula invertida en apoyo del proceso de formación profesional del psicólogo
}

\section{Web 2.0 resources applied in the classroom inverted in support of the process of professional training of the psychologist}

\author{
Néstor Fernández Sánchez ${ }^{1}$ \\ https://orcid.org/0000-0002-6100-5855 \\ Universidad Nacional Autónoma de México \\ Paola Alexandra Quispe Palpa ${ }^{2}$ \\ Universidad Nacional Mayor de San Marcos, Perú
}

Recibido: 13-04-2019

Aceptado: 31-07-2019

\section{Cita Recomendada}

Fernández, N. \& Quispe, P. (2019). Recursos de la Web 2.0 aplicados en el aula invertida en apoyo del proceso de formación profesional del psicólogo. Hamut'ay, 6(2), 69-84.

http://dx.doi.org/10.21503/hamu.v6i2.1776

\section{RESUMEN}

El objetivo de este estudio fue favorecer el aprendizaje de los conceptos relacionados con los primeros días de la vida, desde la perspectiva de la Psicología, en un grupo de 114 estudiantes de la carrera de psicología, aprovechando principios metodológicos del aula invertida, bajo la hipótesis de que el aprendizaje relacionado con los primeros días de la vida, desde la perspectiva de la psicología se verá favorecido al aplicar estrategias de enseñanza derivadas de la propuesta Aula invertida. El estudio se desarrolló bajo el método cuasiexperimental pretest-postest, con una muestra asignada por conveniencia, en el que se aplicó un cuestionario elaborado ad hoc para explorar los conocimientos previos y posteriores a un curso. La diferencia de 53.76 ( $\mathrm{p}<0.001$, con T-Student) entre las calificaciones iniciales y finales generales, así como en cada tema específico permiten confirmar que la forma de trabajo realizada bajo los principios de Aula invertida benefició el aprendizaje de los conceptos deseados.

Palabras Clave: aula invertida, aprendizaje autorregulado, enseñanza centrada en el estudiante.

\section{Abstract}

The objective of this study was to favor the learning of the concepts related to the first days of life, from the perspective of Psychology, in a group of 114 psychology's students, taking advantage of me-

\footnotetext{
${ }^{1}$ Licenciado y maestro en Psicología; doctor en Pedagogía. Profesor de tiempo completo en la Facultad de Psicología de la Universidad Nacional Autónoma de México. Formador de formadores y asesor en la planeación, desarrollo y evaluación de Ambientes Virtuales de Aprendizaje. Líneas de investigación: desarrollo humano, capacitación y educación a distancia. E-mail: nnffss@gmail.com

${ }^{2}$ Estudiante de Psicología de la Universidad Nacional Mayor de San Marcos, Perú. Participante del programa de movilidad en la Facultad de Psicología de la Universidad Nacional Autónoma de México. Líneas de investigación: Desarrollo humano, capacitación, aprendizaje en dominios cognoscitivos y complejos. E-mail: depao1721@gmail.com
} 
thodological principles of the Flipped classroom, under the hypothesis that the learning related to the first days of life, from the psychology's perspective will be favored when applying teaching strategies derived from the Flipped classroom. The study was developed under the quasi-experimental method pretest-postest, with a sample assigned for convenience, in which a questionnaire developed adhoc was applied to explore the knowledge before and after a course. The difference of 53.76 (p $<0.001$, with T-Student) points between the initial and final general grades, as well as in each specific subject, confirm that the way of working carried out under the Flipped classroom benefited the learning of the desired concepts.

Keywords: flipped classroom, self-regulated learning, student-centered teaching.

\section{INTRODUCCIÓN}

En respuesta a diferentes necesidades que presentan diversos países para su desarrollo, las Instituciones de Educación Superior se esfuerzan por formar profesionistas en diversas disciplinas; cada una de ellas, por medio de planes de estudio que atienden aspectos teóricos, prácticos y éticos que favorecen la instauración de competencias que responden al entorno en el que se desempeñarán los futuros agentes participativos en la sociedad.

El presente estudio se enfoca en la formación del psicólogo en la Facultad de Psicología de una universidad pública en México, cuyo perfil de egreso comparte con más de las 290 escuelas o facultades que forman licenciados en esta disciplina, formación como señalan Carlos \& Núñez (2008) orientada a la atención en el comportamiento de los humanos como destinatarios del servicio profesional, coincidiendo así, con el objeto de estudio: el comportamiento humano, independientemente del enfoque teórico-metodológico que sustenta ese plan de estudios. Y, para comprender éste, es necesario analizar las características y factores de influencia en el desarrollo de las personas.

Toda vez que el tema principal, es la persona como parte de una sociedad y como individuo, la asignatura denominada "Ciclo de vida", (Desarrollo psicológico, Desarrollo humano, Desarrollo evolutivo, Psicología evolutiva, etc.) juega un papel de suma importancia en la formación de estos profesionistas. En el estudio que se presen- ta, la asignatura con objetivo general el "construir una visión integral y completa de los principales hitos dentro del ciclo de vida del desarrollo humano, considerando los factores de influencia y el papel del psicólogo en este proceso", según expone la Facultad de Psicología (Ciclo de vida, 2011). En la descripción del programa educativo, se propone una serie de estrategias de enseñanza para lograr los objetivos de aprendizaje, los cuales pretenden evitar la forma expositiva del predominio en la promoción del aprendizaje enciclopédico, en el proceso educativo de la formación profesional, de acuerdo con Carlos \& Núñez (2008) y Carlos \& Guzmán (2016), al mismo tiempo que se busca favorecer el aprendizaje, orientando la práctica docente hacia la atención del proceso de enseñanza centrada en el estudiante, donde las adopciones o maneras particulares y características de pensar el proceso educativo y de asumir el proceso de enseñanza se dé en función de diversos aspectos: contexto, relación, interacción, socialización y orientación de los estudiantes. También la organización, preparación o planificación de la actividad académica; presentación o distribución de la información; así como métodos de enseñanza, aprendizaje, dirección de las tareas y evaluación. Dichas afirmaciones han sido expuestas por McCombs \& Whisler (1997); Díaz Hernández (2010) y Rendón (2010).

Cabe aclarar que el interés de la presente investigación no se orienta hacia un problema relacionado con el aprendizaje de los estudiantes, pues 
no hay evidencia documentada acerca de índices de reprobación o desaprovechamiento escolar, derivado de las actuales formas de proceder en la asignatura por los diversos profesores. En reuniones de trabajo entre éstos se ha identificado la preocupación por atender de manera efectiva a los estudiantes, por medio de formas de trabajo innovadoras que propicien la calidad del aprendizaje. Se pretende con lo anterior promover que el estudiante sepa pensar al comenzar la actividad y durante la realización de la misma; así como saber hacer uso de los contenidos revisados al finalizar el acto educativo, favoreciendo el aprendizaje autorregulado, de acuerdo con las ideas expuestas originalmente por Monereo (1990) y retomadas por otros estudiosos como Costa \& García (2017) y Díaz et al., (2017). Se rescatan, además, las ideas de Torrano, Fuentes \& Soria (2017) quienes expusieron que el aprendizaje es más efectivo cuando supone una construcción significativa a partir de las experiencias y el conocimiento previo, las cuales dotan al aprendiz de diferentes estrategias, capacidades y perspectivas hacia el aprendizaje.

Este aprendiz buscará crear representaciones congruentes y significativas del conocimiento, relacionará significativamente la información nueva con la ya existente, y construirá y utilizará una serie de estrategias de pensamiento y razonamiento para alcanzar las metas de aprendizaje (Coll \& Monereo, 2008).

Como parte de los diversos contenidos temáticos que se abordan en la asignatura, se observa para el presente estudio el de "Primeros días de la vida", que pertenece a la Unidad denominada "De la concepción a la adolescencia". Con base a las propuestas de estrategias de enseñanza, los mecanismos para la evaluación y la orientación del aprendizaje centrada en el estudiante, se adoptaron los principios del Aula Invertida para el desarrollo de éste y otros temas de la asignatura.

En congruencia con los objetivos de la asignatura, para evaluar el aprendizaje de los estudiantes, se elaboró un cuestionario exploratorio tomando en cuenta las dimensiones del conocimiento de Bloom que adoptó originalmente Churches (2008) y retoman Taquez, Rengifo \& Mejía (2017) para diseñar un instrumento de evaluación.

\section{Tabla 1}

Dimensiones conocimiento Bloom para diseño de instrumento de evaluación

\begin{tabular}{ll}
\hline Conocimiento & Recordar información previamente aprendida \\
\hline Comprensión & $\begin{array}{l}\text { La habilidad de entender el significado de la } \\
\text { información. }\end{array}$ \\
\hline Aplicación & $\begin{array}{l}\text { La habilidad de usar la información aprendida } \\
\text { en una situación nueva y específica. Los pro- } \\
\text { cesos implicados son la aplicación de reglas, } \\
\text { métodos, conceptos, principios, leyes y teorías. }\end{array}$ \\
\hline Análisis & $\begin{array}{l}\text { La habilidad de descomponer el todo en sus } \\
\text { partes para su distinción, clasificación y rela- } \\
\text { ción en la solución de problemas }\end{array}$ \\
\hline Síntesis & $\begin{array}{l}\text { La habilidad de resolver contradicciones y re- } \\
\text { estructuras las partes en un nuevo todo. Se } \\
\text { crea aplicando el conocimiento y habilidades } \\
\text { anteriores para producir algo nuevo u original. }\end{array}$ \\
\hline Evaluación & $\begin{array}{l}\text { La habilidad de juzgar el valor de información } \\
\text { compilada para un propósito en específico. Es } \\
\text { emitir juicios sobre la base de criterios prees- } \\
\text { tablecidos }\end{array}$ \\
\hline
\end{tabular}

Fuente: Churches, 2008; Taquez, Rengifo \& Mejía, 2017.

La impartición de la asignatura, como otras del plan de estudios, se realiza por medio de la "clase" tradicional. En esta ocasión se intentó probar el Aula invertida como una opción más para favorecer el aprendizaje. Por lo que en este estudio se tuvo como objetivo incrementar el aprendizaje de los conceptos relacionados con los primeros días de la vida, desde la perspectiva de la Psicología, en un grupo de estudiantes de la carrera de psicología, aprovechando principios metodológicos del aula invertida. Y como Hipótesis: El aprendizaje de conceptos relacionados con el tema "Los primeros días de la vida", desde la perspectiva de la psicología, en un grupo de estudiantes de la carrera de psicología, se verá incrementado al aplicar estrategias de enseñanza derivadas de la propuesta Aula invertida.

\section{Aula invertida}

La propuesta de Aula invertida surge en el 2000 con las ideas de Maureen Lage y sus colaboradores, así como por los trabajos de Crouch \& Mazur (2001); la forma de trabajo se popularizó con la difusión de las experiencias de Bergmann \& Sams (2012) quienes, en pocas palabras, decidieron distribuir el conocimiento que debería aprender 
el estudiante por medio de las explicaciones que éstos hacían normalmente en sus clases, ahora por medio de videos y cambiando el orden de la sesiones de clase; una vez que el estudiante revisaba a voluntad y en sus tiempos disponibles dichos contenidos, durante la clase se dedicaron a promover la realimentación entre los propios estudiantes con asistencia del docente. En otras palabras, se invirtió el orden en la forma de trabajo.

La propuesta de Aula invertida intenta romper paradigmas clásicos del proceso de enseñanza escolarizado, consciente de que en la mayoría de las instituciones de educación el escenario planeado para el ejercicio del proceso de enseńanza es el aula, parte de la infraestructura de las escuelas para que el profesor pase al frente y "dé la clase" o "imparta la cátedra”. Quienes han tenido la oportunidad de asistir a la escuela, saben que los docentes tienen diversas formas de trabajo: la exposición libre, la declaración escrita de contenidos en el pintarrón, la presentación de imágenes y la combinación de éstas, entre otras. En estas condiciones, el profesor es la figura central y los estudiantes asumen su rol receptor al escuchar y rescatar las ideas mediante la "toma de apuntes" que declara el experto. Aquellos profesores que han identificado las benevolencias del uso de algunos recursos que ofrecen las Tecnologías de la Información y la Comunicación (TIC) apoyan su exposición por medio de representaciones gráficas - fijas o en movimiento - con apoyo de un proyector. El proceso esperado, típico, es que el docente asigne tareas a realizar en casa al finalizar la lección.

La mayoría de los sistemas escolarizados están enfocados a la práctica comentada y si el profesor se percata que algunos alumnos no comprendieron la clase del día, podría atender brevemente los contenidos de ésta para la próxima ocasión en la que asista al aula. Lamentablemente, los programas de estudio están organizados para atender un número específico de objetivos y temas bajo el calendario escolar, no hay tiempos extra clase para aportar asesoría o retroalimentación que disipe las posibles dudas. En la siguiente clase, el profesor revisa el nuevo tema y compila las tareas realizadas por sus pupilos para asignar la calificación respectiva bajo la idea de que la acumulación de buenas calificaciones en dicha práctica dará lugar al aprendizaje esperado, estimado por medio de uno o varios exámenes escritos; en caso de detectar una frecuencia alta de errores en las tareas entregadas podrá retomar el tema, pero no profundizar porque hay mucho material por cubrir antes del examen final. Si los estudiantes comprenden lo pretendido, será exitoso el resultado de dicho examen, caso contrario, queda bajo la responsabilidad de ellos disipar las dudas y estudiar a fondo sus apuntes para lograr una buena calificación.

Afortunadamente, algunos profesores asumen que el elemento principal del proceso educativo es el estudiante y lo importante es el aprendizaje, más que la calificación. Por ello es que se han propuesto diversas formas de trabajo en el aula, apoyándose en estrategias de enseñanza propuestas por Gonzáles (2015) que implican aspectos y fundamentos del enfoque cognoscitivista del aprendizaje y retoman Caira, Urdaneta \& Mata (2014), así como Marqués (2013). En el caso que nos ocupa, se rescata el modelo denominado Aula invertida que consiste en trasladar una parte o la mayoría de la instrucción directa al exterior del aula para aprovechar el tiempo en clase en propiciar la interacción entre profesor y estudiante con el fin de facilitar el aprendizaje.

Los cuatro pilares del Aula Invertida expuestas por Bergmann \& Sams (2012) y Bauer (2016).

Tabla 2

Pilares del Aula invertida

\begin{tabular}{|c|c|}
\hline $\begin{array}{l}\text { Flexible Environ- } \\
\text { met (ambientes } \\
\text { flexibles). }\end{array}$ & $\begin{array}{l}\text { Los estudiantes pueden elegir cuándo y dónde } \\
\text { aprenden; esto permite flexibilidad en el ritmo } \\
\text { de aprendizaje. }\end{array}$ \\
\hline $\begin{array}{l}\text { Learning Culture } \\
\text { (cultura del } \\
\text { aprendizaje). }\end{array}$ & $\begin{array}{l}\text { Se evidencia un cambio deliberado en la apro- } \\
\text { ximación al aprendizaje de una clase centrada } \\
\text { en el profesor a una en el estudiante. }\end{array}$ \\
\hline $\begin{array}{l}\text { Intentional Con- } \\
\text { tent (contenido } \\
\text { intencional). }\end{array}$ & $\begin{array}{l}\text { Para desarrollar un diseño instruccional apro- } \\
\text { piado hay que hacerse la pregunta: ¿qué } \\
\text { contenido se puede enseñar en el aula y qué } \\
\text { materiales se pondrán a disposición de los es- } \\
\text { tudiantes para que los exploren por sí mismos? }\end{array}$ \\
\hline $\begin{array}{l}\text { Professional } \\
\text { Educator (do- } \\
\text { cente profesio- } \\
\text { nal). }\end{array}$ & $\begin{array}{l}\text { Los docentes definen y adaptan la forma de } \\
\text { atender los contenidos, así como la forma de } \\
\text { instrucción y las opciones para maximizar el } \\
\text { tiempo en la clase; en esta, observa y provee } \\
\text { retroalimentación evaluando permanentemen- } \\
\text { te el trabajo de los estudiantes. }\end{array}$ \\
\hline
\end{tabular}

Fuente: Bauer, (2016) 
Estas aportaciones las retomó Calvillo (2014) y propuso once indicadores de apoyo para el docente que intenta instaurar el Aula invertida como parte de su práctica, tomando los siguientes principios:

- El facilitador motiva a los estudiantes, haciéndoles ver la importancia que tiene el tema a revisar, bajo la sugerencia de organizador anticipado propuesto por David Ausubel, mismo que retoman Moreira (2008) y Woolfolk (2010).

- El facilitador planea propositivamente el cambio en el orden (inversión) de su práctica docente para favorecer el aprendizaje de los contenidos.

- El profesor procura una situación en la que los estudiantes pueden elegir dónde realizar la actividad de aprendizaje, antes de la sesión de clase.

- Las instrucciones y material de apoyo están a disposición de los estudiantes, incluso para los que no asistieron a la sesión en la que se describió el planteamiento de la dinámica a seguir; ello aprovechando los recursos de las TIC expuestas por Vidal et al., (2016).

- El planteamiento de la actividad para el aprendizaje es flexible en términos de la disponibilidad del tiempo para realizarla antes de llegar a la clase.

- El tiempo destinado para el trabajo en el aula se dedica para atender con profundidad aquellos contenidos (declarativos, procedimentales o actitudinales) que el estudiante revisó en la actividad fuera del aula previamente.

- El tiempo destinado para la clase se invierte en la revisión de los contenidos para favorecer la oportunidad de confirmar los aprendizajes pretendidos.

- Durante el tiempo en el aula, el facilitador favorece el trabajo participativo y colaborativo, orientado hacia la verificación de los contenidos del tema a tratar.

- En el trabajo realizado en el aula se promueve la autoevaluación del aprendizaje logrado.

- Los materiales de apoyo para la realización de la actividad de aprendizaje, previa al tiempo destinado a la clase, cuentan con un arreglo didáctico que responde a la intención educativa.

- Durante el tiempo de clase, el facilitador adop- ta métodos o estrategias de enseñanza - más allá de la exposición - para favorecer el aprendizaje significativo y centrado en el estudiante, como las que expusieron Díaz-Barriga \& Hernández (2010).

- El facilitador se mantiene atento para identificar a aquellos estudiantes que puedan manifestar imprecisiones o incertidumbre en el aprendizaje esperado. En aquellos casos que el facilitador identifica imprecisiones o incertidumbre en el aprendizaje esperado, proporciona realimentación. Para el caso, hoy en día se aprovechan diversas opciones de comunicación que nos ofrecen las TIC, como lo expresó García (2015).

- El facilitador es tolerante, pero orientador hacia el aprendizaje pretendido, ante el posible caos que se propicia cuando se favorece el trabajo en equipos durante la clase.

- El facilitador promueve la realimentación de su práctica docente apoyándose en otro(s) facilitador(es) antes o después del ejercicio del aula invertida, según las ideas de Medina (2017).

Experiencias de esta práctica han sido reportadas por Fulton, $(2014,2013)$ en educación media superior, Merla \& Yañez (2016) en docentes de educación media superior, Betihavas et al., (2016) en enfermería, Santiago, Díez \& Andía (2017) en educación superior, Madrid et al., (2018) en matemáticas para bachillerato, Espinosa, Solano \& Veit (2018) en clases de Física, González (2019) en prosumidores de recursos educativos, entre otros. Incluso, se ha probado esta forma de trabajo con alumnos con dificultades de aprendizaje (Peinado, 2018). El tema ha tenido impacto tal, que existen grupos de trabajo que estudian, analizan y aplican el Aula invertida, como Flipped Learning (https:// flippedlearning.org/) y The Flipped Classroom que ofrece certificación para usuarios de la metodología (https://www.theflippedclassroom.es/); por otro lado, recientemente se propuso "Flipped Learning 3.0” por Santiago \& Bergman (2018).

Recursos de la Web 2.0 en apoyo al Aula invertida

Además de "invertir" la secuencia clásica en el proceso de enseńanza, en el Aula invertida se requiere 
la distribución de los materiales a revisar antes de su análisis en el tiempo destinado para la reunión con los estudiantes, motivo de lo anterior es que se aprovechan los recursos TIC, específicamente los recursos de la Web 2.0, para la distribución de los contenidos, instrucciones y entrega de las evidencias de aprendizaje.

Como señalan Fernández \& Godoy (2017), en la actualidad existe una enorme variedad de recursos que permiten dicha distribución por medio de imágenes fijas, textos, audios, videos y combinaciones de estos (ver ejemplos en la tabla 3).

\section{Tabla 3}

Recursos de la Web 2.0 en apoyo al Aula invertida

\begin{tabular}{|c|c|}
\hline \multirow{5}{*}{$\begin{array}{l}\text { Videos interac- } \\
\text { tivos }\end{array}$} & Panopto (www.panopto.com) \\
\hline & Screencast-o-matic (screencast-o-matic.com) \\
\hline & edpuzzle (https://edpuzzle.com) \\
\hline & Screenr (articulate.com) \\
\hline & PlayPosit (go.playposit.com) \\
\hline \multirow[t]{3}{*}{ Murales virtuales } & MURAL (mural.co) \\
\hline & Gloster (edu.glogster.com) \\
\hline & Padlet (es.padlet.com) \\
\hline \multirow[t]{3}{*}{ Presentaciones } & Slideshare (www.slideshare.net) \\
\hline & Photopeach (photopeach.com) \\
\hline & mydocumenta (mydocumenta.com) \\
\hline \multirow{4}{*}{$\begin{array}{l}\text { Cuestionarios } \\
\text { interactivos }\end{array}$} & mydocumenta (mydocumenta.com) \\
\hline & quizbean (quizbean.com) \\
\hline & goconqr (www.goconqr.com) \\
\hline & Hotpotatoes \\
\hline \multirow{6}{*}{$\begin{array}{l}\text { Actividades } \\
\text { individuales y } \\
\text { colaborativas }\end{array}$} & Jclick (clic.xtec.cat) \\
\hline & Cuestionarios de HotPotatoes (hotpot.uvic.ca) \\
\hline & Educaplay (es.educaplay.com) \\
\hline & Google Docs \\
\hline & Líneas de tiempo Visme (www.visme.co) y \\
\hline & Timeline (timeline.knightlab.com) \\
\hline \multirow{4}{*}{$\begin{array}{l}\text { Grabación de } \\
\text { audio y video }\end{array}$} & Audacity (www.audacityteam.org) \\
\hline & Nero Wave Editor (www.nero.com) \\
\hline & Goldwave (www.goldwave.com) \\
\hline & Flipgrid (flipgrid.com) \\
\hline $\begin{array}{l}\text { Comunicación } \\
\text { permanente }\end{array}$ & Redes sociales como WhatsApp y Facebook \\
\hline $\begin{array}{l}\text { Lanzadores de } \\
\text { enlaces }\end{array}$ & $\begin{array}{l}\text { Generadores de QR (the-qrcode-generator. } \\
\text { com o qr-code-generator.com) }\end{array}$ \\
\hline
\end{tabular}

Fuente: Elaboración propia adaptada de AulaPlaneta (2015).
Por supuesto, lo anterior implica que los estudiantes cuenten con Internet, afortunadamente el servicio de esta tecnología se ha incrementado por los usuarios en actividades de apoyo para la formación o la educación, según reporta la Asociación de Internet México (AIMX, 2019).

Cabe señalar la advertencia que hizo el Tecnológico de Monterrey (ITESM, 2014) acerca de que la propuesta de Aula invertida no es un modelo innovador (de hecho, comenta que no es un modelo), pero es interesante tomar en cuenta la propuesta toda vez que se declara un orden y recomendaciones del enfoque centrado en el estudiante y ello requiere una sistematización de la planeación del acto educativo, así como un cambio de paradigma acerca de la práctica docente. En contraposición, Pedro Rut expone en su tesis para obtener el Master en formación de profesorado que la esencia del método es una forma innovadora y efectiva para promover aprendizajes cuando el docente está motivado para innovar; así mismo, el efecto será propiciar la formación de personas creativas que expone Rut, (2017).

Toda vez que los objetivos del programa de la asignatura mencionada en esta investigación, se orientan hacia niveles taxonómicos que van más allá del conocimiento de los contenidos, se contempló, la incursión de una nueva forma de trabajo para favorecer el aprendizaje de los estudiantes, pretendiendo el logro de niveles de comprensión y análisis de los diversos temas, incluso, con la intención de promover en ellos pautas para el aprendizaje autorregulado. De forma paralela, el propósito es describir una opción metodológica de aplicación del aula invertida en el contexto de la educación superior orientada a la formación profesional del psicólogo.

\section{Materiales Y Métodos}

\section{Participantes}

La población estuvo constituida por una muestra por conveniencia (Otzen, Manterola, 2017) integrada por 114 estudiantes de los 145 inscritos originalmente en la asignatura de Ciclo de Vida, del cuarto semestre de la carrera de Psicología de 
la Facultad de Psicología de una Universidad; 31 de ellos decidieron no participar en las actividades desde el inicio del curso. De los 114 participantes, 36 inscritos en el Sistema de Universidad Abierta y 109 en el sistema escolarizado, ambos atendidos de manera semi presencial con apoyo de la plataforma Moodle de la mencionada Facultad de Psicología y por el mismo profesor.

Aunque la participación fue voluntaria, se solicitó el consentimiento de los participantes, garantizándoles la protección de su identidad, en respeto de sus derechos individuales. De esta manera, la información obtenida cuenta con las garantías del caso y los resultados son producto del análisis objetivo y riguroso de los datos.

\section{Instrumento}

Se diseñó un examen de conocimientos a través del Formulario de Google Forms con 34 preguntas cuyas opciones de respuesta se clasificaron en "Afirmativo", "Negativo" o "Duda". Se trata de un extracto del examen general de conocimientos de la asignatura cuya validez de constructo y contenido empírica prevalece desde hace más de 5 años; la confiabilidad es aceptable (Cronbach $\alpha=0.72$ ). En la elaboración ad hoc del instrumento se tomaron en cuenta los seis dominios cognitivos de la Taxonomía de objetivos de la educación para la era digital de Bloom para analizar el nivel de aprendizaje de los ocho temas del programa oficial de la asignatura Ciclo de Vida, de acuerdo con los objetivos expresados en el programa. En el Anexo se muestra un fragmento del instrumento, en el formato original de Google Forms.

\section{Tipo y diseño}

La presente investigación está basada en el diseño cuasi experimental de preprueba - posprueba con un solo grupo en el que se realiza una observación antes y después de una intervención (Segura, 2003). Se consideró para ello como variable dependiente el número de respuestas correctas en el examen exploratorio de conocimientos y la variable independiente fue la intervención educativa los principios de Aula invertida con aplicación de recursos Web 2.0; de manera específica: lectura de códigos $\mathrm{QR}$, solución de cuestionarios soportados en Google Forms, distribución de textos y videos, comunicación por redes WhatsApp y Facebook, localización de documentos con metabuscadores, líneas del tiempo onLine, trabajo colaborativo con Google Docs y evaluación formativa con cuestionario onLine (HotPotatoes).

\section{Procedimiento}

Para la distribución de contenidos, explicación de las actividades a realizar, recepción de evidencias de trabajos elaborados y la asignación de calificaciones se soportó el curso en la plataforma Moodle, versión 3.1.

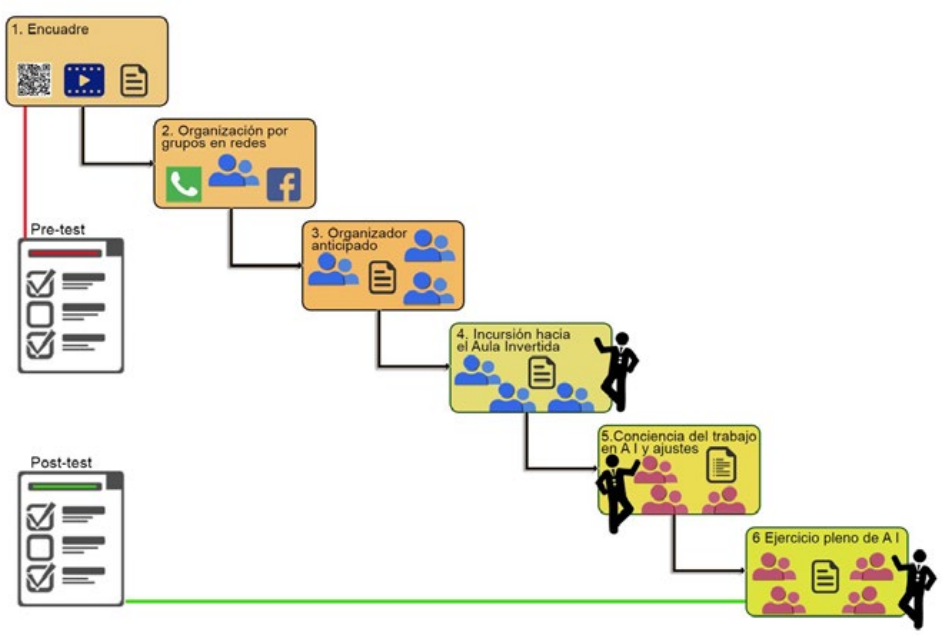

Figura 1

Representación de las fases de intervención.

En la primera sesión de clase, el profesor colocó en las cuatro paredes del aula un código QR que, al leerse, abre el archivo portable (PDF) del programa oficial de la asignatura; este fue el primer acercamiento con la Web 2.0. Se solicitó a quienes tenían el conocimiento y habilidad para abrir el archivo que ayudaran a sus compañeros que no lo tenían. Esta experiencia dio pie para recomendar presentarse a las sesiones de clase con el teléfono celular o Tablet, configurados para conectarse a la Web. De igual forma, se recomendó acceder a la plataforma desde dichos dispositivos tecnológicos.

\section{Fase 1 A. Evaluación inicial.}

La primera actividad formal consistió en atender la sección "Conocimientos previos", con la si- 
guiente instrucción: "Para identificar cuáles son los contenidos temáticos que debemos revisar con mayor atención, se realiza una exploración de sus conocimientos. Se trata de un formulario de Google Forms que integra 34 reactivos representativos de todos los temas del programa, a manera de pre prueba”. El acceso al formulario se determinó desde una liga colocada en la plataforma.

\section{Fase 1 B. Encuadre.}

La siguiente sección expuesta en la plataforma fue el encuadre, que incluyó un análisis del objetivo del programa, la presentación de un mapa conceptual con los temas principales a revisar, la bienvenida (en texto y video), la exposición de la forma de trabajo, formas de evaluación y tres videos, relacionados con las etapas del desarrollo humano, con la intención de reflejar la importancia de comprender las diversas etapas de la vida desde la perspectiva de la psicología y los factores de influencia.

\section{Fase 2. Organización por grupos en redes sociales.}

Antes de atender el siguiente tema, se integraron equipos de 6 participantes aprovechando el recurso "grupos" de la plataforma; se recomendó usar WhatsApp y Facebook a los estudiantes para comunicarse entre sí.

\section{Fase 3. Organizador anticipado hacia la profesión.}

Posterior a la revisión de los videos, se solicitó realizar la actividad denominada "Expectativas sociales". Se distribuyó material de apoyo y ligas en la Web hacia documentos alusivos al tema para identificar la definición de psicología, las sub especialidades en las que puede ejercer el psicólogo y los contenidos que el Centro Nacional de Evaluación para la Educación Superior (CENEVAL) considera en el Examen General para el Egreso de Licenciatura (EGEL) en la carrera de Psicología en México. En el mismo ejercicio se pidió preguntar a dos personas, no estudiantes ni profesores de la carrera, responder a la pregunta ¿Para qué sirven los psicólogos? En el tiempo destinado a clase se realizó un ejercicio, bajo la técnica de cuchicheo (Canarias, 2011), para que los estudiantes intercambiaran ideas acerca de la información obtenida; la participación del profesor consistió en rescatar las aportaciones de los estudiantes, orientando su discurso hacia la importancia de "construir una visión integral y completa de los principales hitos dentro del ciclo de vida del desarrollo humano, considerando los factores de influencia y el papel del psicólogo en este proceso (objetivo del programa de estudio)".

\section{Fase 4. Incursión hacia el aula invertida.}

La siguiente actividad correspondió al tema "Conceptos básicos", en la que cada estudiante debió responder a 17 preguntas relacionadas con los conceptos principales que se manejan en el contexto de la asignatura, basándose en lecturas que se pusieron a disposición en la plataforma y por medio de la localización de documentos en la Web. El resultado de la actividad individual se integró en un ensayo (Olaizola, 2011) que realizaron en equipos de trabajo; además de un resumen individual. Ambas expresiones de elaboración escrita se realizaron en Google Docs. Con lo anterior se pretendió fortalecer e incorporar en los estudiantes el acervo conceptual relacionado con los temas a atender posteriormente. En la sesión de clase se definió cada concepto revisado con la aportación voluntaria de los estudiantes mediante lluvia de ideas (Canarias, 2011); el profesor rescató éstas, las expuso de forma escrita en el pintarrón y comentó la relación que existe entre cada concepto y los diversos temas que se atenderán en posteriores actividades mediante un mapa mental (Flores et al., 2017). Cuando el profesor identificó incertidumbre con relación a la comprensión de algún concepto, sugirió localizarlo en la Web con apoyo del dispositivo móvil; una vez localizado el concepto, el profesor realimentaba la participación y retomaba el tema.

La siguiente actividad correspondió al tema "Perspectivas teóricas". Además de la exposición de una introducción y los objetivos específicos en la plataforma, se entregó una lista con 25 nombres de personajes que han influenciado en el estudio y comprensión del tema Ciclo de vida. La actividad para el aprendizaje consistió en la elaboración de una línea del tiempo (UVM, 2011) en la que los integrantes del equipo incorporaron la imagen representativa del personaje, el nombre completo (la lista tenía el nombre parcial. Como A. Gesell, 
J. Piaget o S. Freud) y las aportaciones principales que identificaron respecto al estudio del ciclo de vida. Para la línea del tiempo se recomendó utilizar el recurso "timelines" en la Web. Para el trabajo en clase se reiteró presentarse con algún dispositivo móvil, como el celular o Tablet. El trabajo en el aula consistió en revisar cada uno de los personajes, con las aportaciones de los estudiantes mediante lluvia de ideas. Cuando se presentaba alguna duda (como en el caso de uno de los personajes que fue confundido con su hijo), el profesor recomendó localizar la información con el buscador de Google. El papel del profesor consistió en retroalimentar las contribuciones, al tiempo que rescataba las mismas y las escribió en el pintarrón.

En este caso se aplicó evaluación formativa, misma que consistió en la resolución de un crucigrama, aprovechando el recurso JCross (Vázquez, Pérez \& Ruíz, 2018) del programa HotPotatoes, con apoyo del celular, Tablet o Laptop; el acceso al crucigrama fue por medio de un código QR distribuido en mano de cada estudiante. En la primera fase de la evaluación, cada estudiante respondió su crucigrama (JCross fue arreglado para que cada visitante tuviera un crucigrama diferente, para cada respuesta incorrecta otorgó ayuda y el tiempo para responder fuera limitado a 10 minutos); en la segunda fase, se solicitó resolver de nuevo el crucigrama, con un solo dispositivo, entre 4 participantes.

\section{Fase 5. Conciencia del trabajo en aula invertida y ajustes.}

Para la siguiente actividad se informó brevemente a los estudiantes en qué consiste la forma de trabajo en aula invertida, principalmente en el cambio de orden de las sesiones de clase; se advirtió que las aportaciones que trajeran a las sesiones serían la parte principal del desarrollo de éstas. De igual forma, se solicitó resolver un cuestionario de opinión de nueve preguntas, en la plataforma, señalando que éste tiene el propósito de promover la eficiencia del proceso de enseñanza en el desarrollo del programa de la asignatura Ciclo de vida, bajo los principios del aula invertida previamente explicado y ejercido.

Las preguntas fueron calificadas por los estudiantes con base a escala de Likert, para opinar acerca de la autonomía (Autonom) y aplicar los estilos de aprendizaje personales. La permisividad para organizar la disponibilidad de sus tiempos en la atención a las actividades recomendadas, la dinámica que se establece en el aula para favorecer el aprendizaje colaborativo (Dinámica), la forma de evaluación (Evaluac), las condiciones para ejercer el estudio independiente (Est-Indep), la comodidad del uso de la plataforma para recibir instrucciones y materiales de apoyo (Plataform), la realimentación del profesor para confirmar el aprendizaje demostrado en los trabajos que se entregan (Realim), el beneficio de la posibilidad de realizar actividades de estudio en su casa (TrabCasa), la forma de organizar los equipos de trabajo por medio de la plataforma (OrganEqu) y el aprovechamiento de la Web para realizar actividades de aprendizaje (UsoTICs).

Los resultados de la encuesta, resuelta por 63 estudiantes, permitió identificar que el principal elemento de opinión favorable es la retroalimentación que el profesor ejerce de forma permanente $(74.6 \%)$; luego, el uso de recursos de la Web para realizar actividades de aprendizaje (63.4); la forma de evaluación, la promoción del estudio independiente, el uso de la plataforma y el trabajo en casa también recibieron - aunque en menor medida que los anteriores aspectos - respuestas favorables $(57.1$, 57.1, 57.1 y 55.6 respectivamente); le siguió el trabajo que promueve la autonomía (46.0), el uso de dinámicas para el trabajo colaborativo en clase (35.1) y, por último la forma de organizar los equipos por medio de la plataforma (26.9). Los comentarios libres (pregunta número 10 , abierta) permitieron abundar en la exploración. Varios estudiantes declararon no estar acostumbrados a trabajar de forma independiente pues la mayoría de sus profesores dan clase "tradicional"; en cuanto a la forma de evaluación, esperaban obtener una calificación numérica, derivada de un examen, en lugar del texto retroalimentador del profesor por el trabajo elaborado. Respecto a la integración de los equipos de trabajo, la mayoría sugirió trabajar con compañeros que ya conocen de semestres anteriores. Con base en lo anterior, en las siguientes actividades se declaró en la plataforma, con mayor detalle, los elementos que se tomarían en cuenta para asignar calificación a los reportes solicitados; además, la 
organización de equipos se reestructuró una vez que los estudiantes se pusieron de acuerdo entre ellos para integrarse.

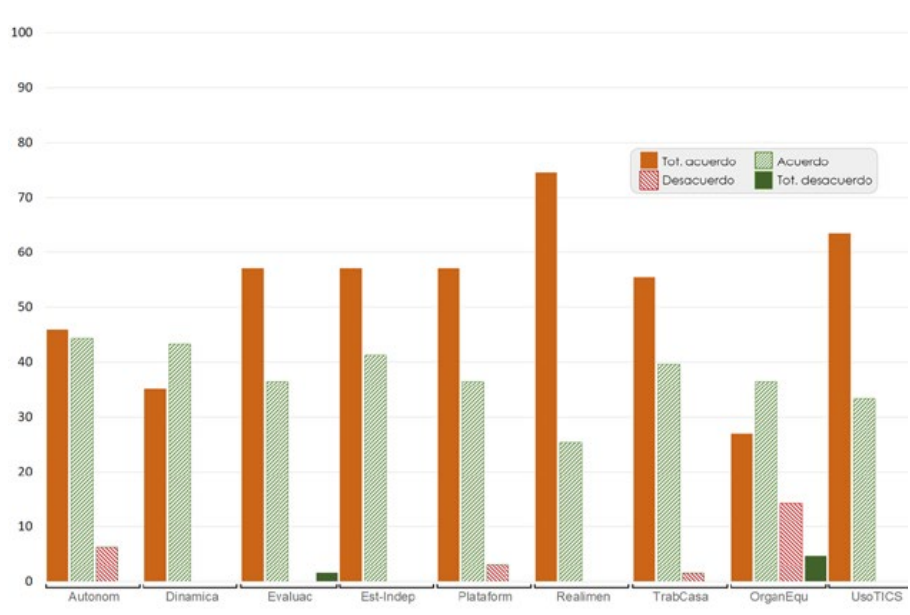

Figura 2

Resultados de la opinión de los estudiantes acerca de la forma de trabajo.

\section{Fase 6. Ejercicio pleno de aula invertida.}

Los siguientes temas para abordar fueron "De la concepción al parto" y "Primeros días de la vida". Como en todos los casos, se expuso en la plataforma una introducción - a manera de organizador anticipado -, los objetivos específicos, las instrucciones de la actividad para el aprendizaje y los materiales de apoyo.

Para el primer tema se solicitó revisar desde la plataforma varios documentos escritos y los videos documentales desarrollados por Discovery History, "El embarazo" para elaborar un tríptico dirigido a padres de familia y cuyo contenido principal fuera la descripción breve del proceso del desarrollo prenatal, los momentos críticos del mismo y los Teratógenos. Es decir, se solicitó realizar una síntesis (UVM, 2011) de los contenidos y la reelaboración de los mismos en un discurso potencialmente aplicado en la sociedad mediante un ensayo (Díaz-Barriga \& Hernández, 2010).

Para el segundo tema, se aprovechó la estrategia de estudio de caso (Stake, 1999), misma que consistió en la presentación de un escenario hipotético en el que se narra la entrevista de los padres de Alberto, inscrito en una estancia de desarrollo infantil, y una psicóloga. El diálogo expuesto expone como problema una serie de términos técnicos que la psicóloga declara y los padres del niño no comprenden (como enuresis, anamnesis, rapport, prueba de Tamiz, Apgar, Brazelton, etc.) y preguntas relacionadas con el tema. Para la narración escrita se aplicaron pistas tipográficas, en color rojo, para los términos importantes, así como 30 preguntas intercaladas, marcadas en azul (Díaz-Barriga \& Hernández, 2010). La actividad para el aprendizaje consistió en elaborar un documento tipo ensayo, hipotéticamente dirigido a los padres de Alberto, para esclarecer los términos técnicos y responder a las preguntas declaradas. Además, debieron presentar la definición técnica en un inventario de términos o glosario (Oliveira, 2013). La sesión de clase consistió, primero, en el intercambio de respuestas al ejercicio; para ello se formaron equipos de 4 o 5 estudiantes con la consigna de despejar dudas entre ellos acerca de contenido de sus reportes. Después de 20 minutos de trabajo de intercambio de ideas, el profesor solicitó exponer las dudas que fueron resueltas, retroalimentó las participaciones estableciendo las relaciones entre los términos analizados y su aplicación en la vida profesional. Para la segunda parte de la sesión, el profesor solicitó responder, en forma individual, las siguientes preguntas: ¿Qué conocimientos nuevos adquirí en este ejercicio? ¿Qué importancia puede tener el conocimiento de este tema y futura vida profesional? y, además de la localización de información para resolver los cuestionamientos ¿cómo fue que aprendí lo que ahora sé de este tema? Después de escuchar algunas de las respuestas, el profesor hizo énfasis en aquellas en las que los estudiantes identificaron sus estilos de aprendizaje, la importancia del trabajo colaborativo y la relación del tema con su vida cotidiana. En consecuencia, se les hizo ver la importancia de asumirse como agentes activos del aprendizaje personal y colectivo.

\section{Resultados}

Después de la primera aplicación del examen de conocimientos se muestra que el grupo de estudiantes de psicología de la asignatura de Ciclo de Vida presentaba conocimientos sobre el tema Primeros años de vida en un promedio bajo de $37.09 \%$. Donde se evidenció un bajo nivel de 
conocimiento en la mayoría de los contenidos a excepción de aquellos representados en los ítems, 17, 16 y 4 (Tabla 3). Cuya mayor prevalencia se explica por la estrecha relación existente entre las materias del plan de estudios de la carrera de Psicología de la UNAM, donde estos contenidos de Ciclo Vida estuvieron presentes en las materias previas revisadas en semestres anteriores, de manera superficial.

\section{Tabla 3}

Resultados de respuestas del Pretest del tema Primeros años de vida)

\begin{tabular}{|c|c|c|}
\hline Ítem y contenido a explorar & $\begin{array}{l}\text { Frecuen- } \\
\text { cia }\end{array}$ & $\begin{array}{l}\text { Porcen- } \\
\text { taje }\end{array}$ \\
\hline $\begin{array}{l}\text { 4. Comprendo la similitud y diferencias } \\
\text { entre Crecimiento y Maduración }\end{array}$ & 101 & 69.66 \\
\hline $\begin{array}{l}\text { 7. Se cuáles son las diferencias y } \\
\text { similitudes entre Crecimiento y } \\
\text { Maduración }\end{array}$ & 45 & 31.03 \\
\hline $\begin{array}{l}\text { 11. Se cuáles son los nombres de } \\
\text { las diferentes etapas en que se } \\
\text { divide el Desarrollo Prenatal y sus } \\
\text { características }\end{array}$ & 24 & 16.55 \\
\hline 12. Sé lo que son los Teratógenos & 22 & 15.17 \\
\hline $\begin{array}{l}\text { 13. Puedo explicar en qué consisten y } \\
\text { para qué sirven la Amniocentesis y } \\
\text { la Prueba de Tamiz }\end{array}$ & 19 & 13.10 \\
\hline $\begin{array}{l}\text { 14. Sé a qué se refieren los teratóge- } \\
\text { nos }\end{array}$ & 25 & 17.24 \\
\hline $\begin{array}{l}\text { 15. Sé a qué se refieren la Amniocente- } \\
\text { sis y la Prueba de Tamiz }\end{array}$ & 26 & 17.93 \\
\hline 16. Puedo explicar lo que es el Apego & 106 & 73.10 \\
\hline $\begin{array}{l}\text { 17. Entiendo la importancia que tiene } \\
\text { la "permanencia del objeto" como } \\
\text { parte del desarrollo del niño }\end{array}$ & 116 & 80.00 \\
\hline
\end{tabular}

Fuente: Elaboración propia (2019).

En los resultados postest (Tabla 4) se muestra que después de la aplicación de Aula Invertida los estudiantes afirmaron que habían comprendido los contenidos correspondientes al tema "Primeros años de vida" en un promedio de $90.84 \%$.

Tabla 4

Resultados de respuestas del Postest del tema Primeros Años de Vida

\begin{tabular}{lcc}
\hline Número de İtem & Frecuencia & Porcentaje \\
\hline İtem 4 & 111 & 97.37 \\
Ítem 7 & 94 & 82.46 \\
Ítem 11 & 101 & 88.60
\end{tabular}

\begin{tabular}{lcc}
\hline Número de Ítem & Frecuencia & Porcentaje \\
\hline Ítem 12 & 105 & 92.11 \\
Ítem 13 & 93 & 81.58 \\
Ítem 14 & 105 & 92.11 \\
Ítem 15 & 99 & 86.84 \\
Ítem 16 & 111 & 97.37 \\
Ítem 17 & 113 & 99.12 \\
\hline
\end{tabular}

Fuente: Elaboración propia (2019).

En lo correspondiente a la media de los resultados pretest con la media de los resultados postest se encuentra que, con respecto al $37.09 \%$ del primero, se mostró un aumento de los conocimientos en el tema de "Primeros años en Vida" en un $53.75 \%$. Evidenciándose que se pasó de una media baja significativamente a una media alta significativamente. Las varianzas de las calificacones pre y post que se muestran en la figura 3 son también una evidencia del cambio tan importante que se produjo en la experiencia.

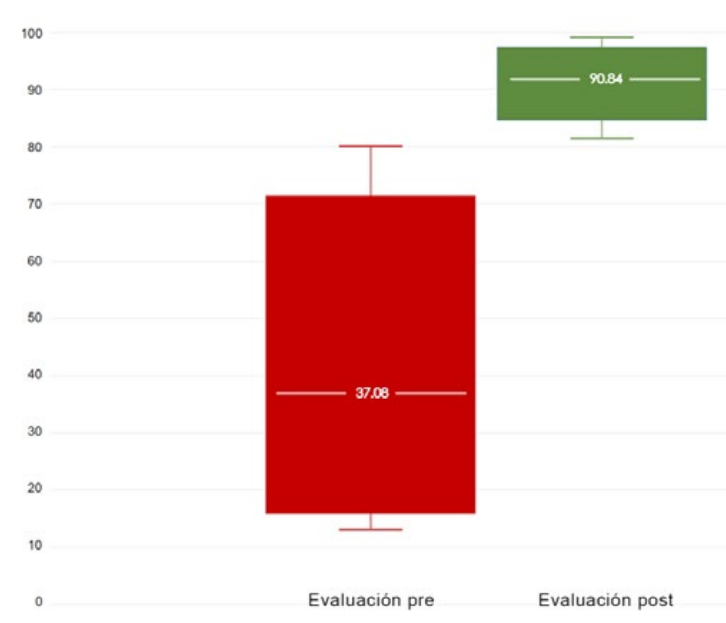

Figura 3

Promedios y varianzas de las calificaciones Pretest y Postest para la exploración de conocimientos de Primeros Años de Vida.

Se muestra que en el ítem 13, donde el resultado pretest tuvo el menor valor, pudo elevarse el mismo hasta en un 81.58, mostrándose que se pudo maximizar el conocimiento en aquel contenido por el uso del Aula Invertida. Por otro lado, se evidencia que en los ítems 4, 16 y 17 no se encontró una alta diferencia entre el pretest y el postest, (Tabla 5) al ser temas ya tocados en asignaturas anteriores, sin embargo, se observa que es en estos ítems donde se denota que hubo los más altos 
porcentajes pudiendo implicar que gracias al Aula Invertida estos contenidos del tema fueron aprehendidos con mayor nivel. Asimismo, se muestra que en el ítem 12 se halló la mayor varianza entre resultado pretest y postest, con lo que se denota que las estrategias usadas para tal contenido fueron efectivas.

\section{Tabla 5}

Comparación de resultado Pretest y Postest del tema Primeros Años de Vida

\begin{tabular}{lccc}
\hline Número de Ítem & $\begin{array}{c}\text { Pretest } \\
\%\end{array}$ & $\begin{array}{c}\text { Postest } \\
\%\end{array}$ & $\begin{array}{c}\text { Diferencia de } \\
\text { porcentajes }\end{array}$ \\
\hline Ítem 4 & 69.66 & 97.37 & 21.71 \\
Ítem 7 & 31.03 & 82.46 & 51.43 \\
Ítem 11 & 16.55 & 88.60 & 72.05 \\
Ítem 12 & 15.17 & 92.11 & 76.94 \\
Ítem 13 & 13.10 & 81.58 & 68.48 \\
Ítem 14 & 17.24 & 92.11 & 74.87 \\
Ítem 15 & 17.93 & 86.84 & 68.91 \\
Ítem 16 & 73.10 & 97.37 & 24.27 \\
Ítem 17 & 80.00 & 99.12 & 19.12 \\
\hline Todos & 37.08 & 90.84 & 53.76 \\
\hline
\end{tabular}

Fuente: Elaboración Propia (2019)

\section{Prueba T}

El valor de p para la comparación de medias en la tabla 5 es de .001 . Muestra que sí hay una diferencia significativa entre los resultados pretest $y$ los resultados postest del grupo de estudiantes de Psicología de la asignatura Ciclo de Vida.

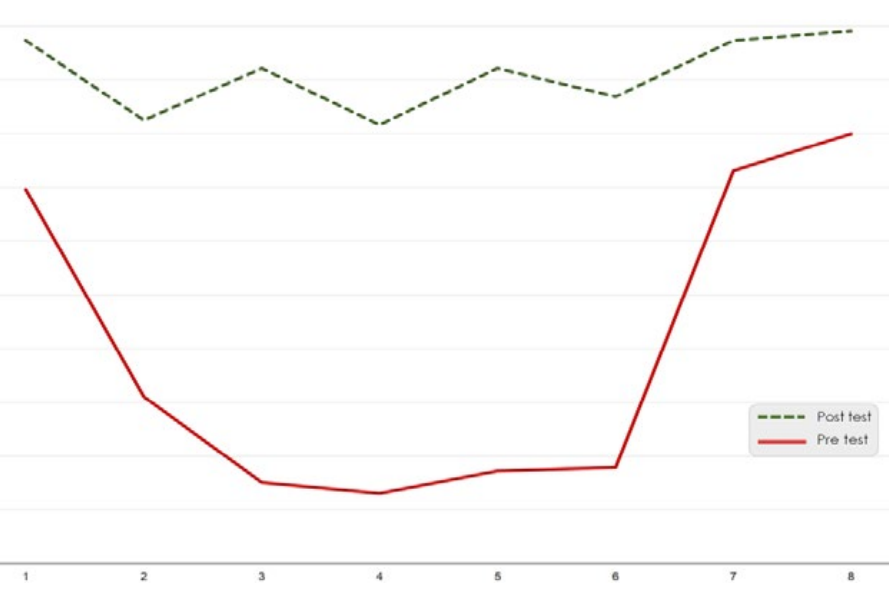

Figura 4

Comparación de resultados Pretest y Postest, tema Primeros Ańos de Vida, por reactivo.

\section{Discusión y Conclusiones}

Con base a los resultados expuestos, se confirma, en términos generales, que el aprendizaje relacionado con el tema "Los primeros días de la vida", desde la perspectiva de la psicología, en un grupo de estudiantes de la carrera de psicología, incrementó al aplicar estrategias de enseñanza derivadas de la propuesta Aula invertida.

Es menester separar los datos de los estudiantes del Sistema de Universidad Abierta, ya que las sesiones clase se realizaron sólo con algunos de ellos, por videoconferencia; las condiciones no fueron iguales para todos.

Es posible que las diferencias significativas puedan no atribuirse al trabajo bajo la perspectiva de aula invertida y solo la estrategia de estudio de caso fuera suficiente. No obstante, el orden de secuencia para atender el caso resultó provechoso.

Además de un estudio más controlado, bajo la perspectiva cuasi experimental, será provechoso combinar con un estudio longitudinal para evaluar, si el aprendizaje se aplica en contenidos a revisar en asignaturas de semestres posteriores. Otra forma de confirmar la eficiencia del método descrito sería aplicar un grupo control y otro experimental, como ya lo hizo en este tema de Aula invertida Enrique Sánchez y sus colaboradores (Sánchez, R. E., Sánchez, R. J. \& Ruíz, 2019).

Otro punto a tomar en cuenta es el posible inconveniente del uso del método ante alumnos que cuentan con una larga historia (y cultura) de trabajo en clase tradicional, en la que la pasividad atrae un estado de confort orientado a recibir del docente los conocimientos que requiere; de otra forma, puede también estar esperando cumplir los requisitos típicos de una presentación de examen en el que demuestre su capacidad memorística, como señalan Aguilera et al., (2017).

Por supuesto, - como indica Pedro López, de la Universidad de la Rioja - este tipo de formas de trabajo requieren que los actores del proceso educativo cuenten con los dispositivos tecnológicos respectivos y el acceso a Internet (López et al., 2016); no en todos los ciudadanos o centros escolares se tiene acceso a ello (Martínez, 2018). y 
algunos docentes aún no se habilitan en el uso de las TIC.

Si bien es cierto que la validez del cuestionario pudiera ser dudosa, ésta se respalda en la experiencia del profesor responsable de su elaboración ya que cuenta con más de 30 años impartiendo la asignatura y evaluando a los estudiantes en exámenes ordinarios y extraordinarios. Parte del instrumento fue elaborado rescatando exámenes previamente aplicados.

\section{REFERENCIAS BIBLIOGRÁFICAS}

Aguilera, R. C.; Manzano, L.; Martínez, M. I; Lozano, S. M. \& Casiano, Y. C. (2017). El modelo Flipped Classroom. International Journal of Developmental and Educational Psychology, 4,1, 261-266. https://doi.org/10.17060/ijodaep.2017.n1.v4.1055

AIMX-Asociación de Internet de México, (2019). Actividades en línea vs. Presenciales. $15^{\circ}$ Estudio sobre los hábitos de los usuarios de Internet en México. Reporte de la Asociación de Internet MX. México.

AulaPlaneta, (2015). 40 herramientas para aplicar la metodología flipped classroom en el aula. Recuperado de https:// www.aulaplaneta.com/2015/05/12/recursos-tic/40-herramientas-para-aplicar-la-metodologia-flipped-classroom-en-el-aula-infografia/

Bauer, K. (2016). Clase invertida. Reseńa. Revista Innovación educativa con TI, julio. Coordinación general de tecnologías de información, Universidad de Colima. Recuperado de https://portal.ucol.mx/content/micrositios/260/file/claseinvertida_resena.pdf

Bergmann, J. \& Sams, A. (2012). Flip your classroom. Reach every student in every class every day. Estados Unidos: International Society for Technology in Education.

Betihavas, V., Bridgman, H, Kornhaber, R. \& Cross, M. (2016). The evidence for "flipping out": A systematic review of the flipped classroom in nursing education. Nurse Education Today, 38, 15-21. https://doi.org/10.1016/j. nedt.2015.12.010

Caira, R. J., Urdaneta E. M. \& Mata G. L. (2014). Estrategias para el aprendizaje significativo de procesos de fabricación mediante orientación constructivista. Revista Opción, 30, 75, $92-103$.

Calvillo, C. A. (2014). El modelo Flipped Learning aplicado a la materia de música en el cuarto curso de educación secundaria obligatoria: una investigación-acción para la mejora de la práctica docente y del rendimiento académico del alumnado. (Tesis doctoral). España: Universidad de Valladolid.
Canarias, G. (2011). Dinámicas de grupo. Materiales para dinámicas de grupos. Área de Tecnología Educativa Medusa, CEP Norte de Tenerife, España: Gobierno de Canarias.

Carlos G. J. \& Guzmán R. M. (2016). Estrategias y métodos para enseñar contenidos psicológicos. Proyecto PAPIME 309804, Universidad Nacional Autónoma de México, Facultad de Psicología.

Carlos, G. J. \& Núnez, C. P. (2008). Análisis curricular de la formación del psicólogo en México. Capítulo del libro del libro Carlos, J. (coord.), La enseñanza de la psicología. Aportaciones de la investigación para mejorar la docencia teórica-práctica.

Churches, A. (2008). Taxonomía de Bloom para la era digital. Recuperado de http://eduteka.icesi.edu.co/pdfdir/TaxonomiaBloomDigital.pdf

Ciclo de vida, (2011). Programa de asignatura Ciclo de vida, clave 1301. Plan de estudios 2008 de la Facultad de Psicología de la Universidad Nacional Autónoma de México.

Coll, C. \& Monereo, C. (2008). Psicología de la educación virtual. Madrid: Editorial Morata.

Costa, C. R. \& García, G. O. (2017). El aprendizaje autorregulado y las estrategias de aprendizaje. Revista Tendencias pedagógicas, 30, 117-130.

https://doi.org/10.15366/tp2017.30.007

Crouch, C. H. \& Mazur, E. (2001). Peer Instruction: Ten years of experience and results. American Association of Physics Teachers, 69, (9), 970-977.

https://doi.org/10.1119/1.1374249

Díaz, M. A., Pérez VM., González, P. J., Núńez, P. J. (2017). Impacto de un entrenamiento en aprendizaje autorregulado en estudiantes universitarios. Perfiles Educativos, XXXIX, 157, 87- 104.

Díaz-Barriga, A. F. \& Hernández, R. G. (2010). Estrategias docentes para un aprendizaje significativo. Una interpretación constructivista. México: Editorial McGraw-Hill.

Espinosa, T., Solano, A. \& Veit, E. (2018). Aula invertida (flipped classroom): innovando las clases de física. Revista de enseñanza de la Física, 30, 2.

Fernández, M. \& Godoy, G. (2017). Aula Invertida para la inclusión de Recursos Educativos Abiertos. IV Workshop Recursos Educativos Abiertos. 12 de octubre, La Plata, Argentina.

Flores, F. J., Ávila A. J., Rojas J. C., Sáenz G. F., Acosta T. R. \& Díaz L. C. (2017). Estrategias didácticas para el aprendizaje significativo en contextos universitarios. Chile: Unidad de Investigación y Desarrollo Docente, Universidad de Concepción.

Fulton, K. (2013). Byron's flipped classrooms. District replaces textbooks with a digital approach to instruction. Education Digest, 79, 1, 22-26.

Fulton, K. (2014). Time for Learning: Top 10 reasons why Flipping the Classroom can change education. California, Estados Unidos: Corwin a Sage Company. https://doi.org/10.4135/9781483332772 
García, J. E. (2015). La evaluación del aprendizaje: de la retroalimentación a la autorregulación. El papel de las tecnologías. Revista Electrónica de Investigación y Evaluación Educativa, 21, 2. Universidad de Sevilla, España.

https://doi.org/10.7203/relieve.21.2.7546

González, F. M. (2019). Experiencia del aula invertida para promover estudiantes prosumidores del nivel superior. RIED, Revista Iberoamericana de Educación a Distancia, 22, 2. https://doi.org/10.5944/ried.22.2.23065

Gonzáles, L. F. (2015). Estrategias de enseñanza que desarrollan procesos cognitivos en el área de ciencia, tecnología y ambiente de los docentes del tercer grado de secundaria, UGEL 04 -Trujillo. (Tesis de maestría). Universidad Peruana Cayetano Heredia. Perú.

ITESM (2014). Aprendizaje invertido. México: Instituto Tecnológico y de Estudios Superiores de Monterrey. Reporte EduTrends. Observatorio de innovación educativa.

Lage, M., Platt, G. \& Treglia, M. (2000). Inverting the classroom: A gateway to creating an inclusive learning environment. The Journal of Economic Education, 31 (1), 3043. https://doi.org/10.1080/00220480009596759

López, S. P. (2016). El Flipped Classroom (Aula invertida) y su eficacia en la acción educativa. Trabajo fin de máster, Universidad Internacional de La Rioja. Espańa: Zaragoza.

Madrid, G. E., Ángulo, A. J., Prieto, M. M., Fernández, N. M. \& Olivares, C. K. (2018). Implementación de aula invertida en un curso propedéutico de habilidad matemática en bachillerato. Revista Apertura, 10, 1. México, Guadalajara.

Marqués, G. P. (2013). Nuevas metodologías docentes para mejorar la formación y los resultados académicos de los estudiantes. Padres y Maestros, 351, 16-22. Facultad de Ciencias Humanas y Sociales. España: Universidad Pontificia Comillas.

Martínez, L. A. (2018). En México hay 79.1 millones de usuarios de Internet. El economista, 17 de mayo. México.

McCombs, B. \& Whisler, J. S. (1997). La clase y la escuela centrada en el aprendiz. Estrategias para aumentar la motivación y el rendimiento. México: Editorial Paidós.

Medina, M. J. (2017). La docencia universitaria mediante el enfoque del aula invertida. Barcelona, España: Ediciones Octaedro.

Merla, G. A. \& Yañez E. C. (2016). El aula invertida como estrategia para mejora del rendimiento académico. Revista de Bachillerato a distancia, 16.

https://doi.org/10.22201/cuaed.20074751e.2016.16.57108

Monereo, C. (1990). Las estrategias de aprendizaje en la Educación formal: enseñar a pensar y sobre el pensar. Revista Infancia y aprendizaje, 50, 3-25.

https://doi.org/10.1080/02103702.1990.10822263

Moreira, M. (2008). Organizadores previos y aprendizaje significativo. Revista Chilena de Educación Científica, 7, 2, 23-30.

Olaizola, A. (2011) El ensayo como herramienta en la enseñanza y el aprendizaje de la escritura académica. Reflexión Académica en Diseño y Comunicación, XVI, 61-66. Bue- nos Aires, Argentina.

Oliveira, D. (2013). El Glosario como propuesta didáctica en el aprendizaje de léxico. Revista Nebrija de lingüística aplicada a la enseñanza de lenguas, 13. Universidad Nebrija.

Otzen, T. \& Manterola, C. (2017). Técnicas de muestreo sobre una población a estudio. Revista Internacional de Morfología, 35, 1, 227-232.

https://doi.org/10.4067/S0717-95022017000100037

Peinado, R. P. (2018). La Clase Invertida: una experiencia con alumnos con dificultades de aprendizaje. Tesis de doctorado, Programa Interuniversitario de Doctorado en Tecnología Educativa. España: Universidad de Murcia.

Rendón, M. A. (2010). Los estilos de enseñanza en la Universidad de Antioquia. Primera fase Facultad de Educación. Colombia: Revista Unipluriversidad, 1 (10), 13 - 29.

Rut, S. P. (2017). Aula invertida, metodología del siglo XXI. Memoria del Trabajo Final de Master. Espańa: Universidad de las islas Baleares.

Sánchez, R. E., Sánchez, R. J. \& Ruíz, P. J. (2019). Percepción del alumnado universitario respecto al modelo pedagógico de clase invertida. Magis, Revista Internacional de Investigación en Educación, 11 (23), 151-168.

https://doi.org/10.11144/Javeriana.m11-23.paur

Santiago, R. \& Bergman, J. (2018). Aprender al revés: Flipped Learning 3.0 y metodologías activas en el aula. España: Paidós.

Santiago, R., Díez, A. \& Andía, L. (2017). Flipped classroom: 33 experiencias que ponen patas arriba el aprendizaje. España: Editorial UOC, SL, Colección Outer Edu.

Segura, C. A. M. (2003). Diseños cuasiexperimentales. Facultad Nacional de Salud Pública, Universidad de Antioquia. Colombia. Recuperado de https://bit.ly/2e5FEFD.

Stake, R. E. (1999). Investigación con estudio de casos. Madrid: Ediciones Morata.

Taquez, H., Rengifo, D. \& Mejía, D. (2017). Diseño de un instrumento para evaluar el nivel de uso y apropiación de las TIC en una institución de educación superior. Encuentro Virtual Educa. 13-16 de junio, Bogotá, Colombia.

Torrano, F., Fuentes, J. L. \& Soria, M. (2017). Aprendizaje autorregulado: estado de la cuestión y retos psicopedagógicos. Perfiles Educativos, XXXIX, 156, 160-173. https://doi.org/10.22201/iisue.24486167e.2017.156.58290

UVM (2011). Estrategias de enseñanza aprendizaje. México. Dirección de Innovación y Tecnología Educativa, Coordinación de Educación a Distancia, Universidad del Valle de México.

Vázquez, G. J, Pérez, R. J. \& Ruíz, C. N. (2018). JCross, elaboración de crucigramas para evaluación. III Congreso Internacional de Innovación Educativo. México: Instituto Politécnico Nacional.

Vidal, L. M., Rivera, M. N., Nolla, C. N., Morales, S. I. \& Vialart, V. M. (2016). Aula invertida, nueva estrategia didáctica. Revista Educación media superior, 30, 3. Cuba, La Habana. 
Néstor Fernández Sánchez y Paola Quispe Palpa

Woolfolk A. (2010). Psicología educativa (11a edición). México: Editorial Pearson Educación. 


\section{Anexo}

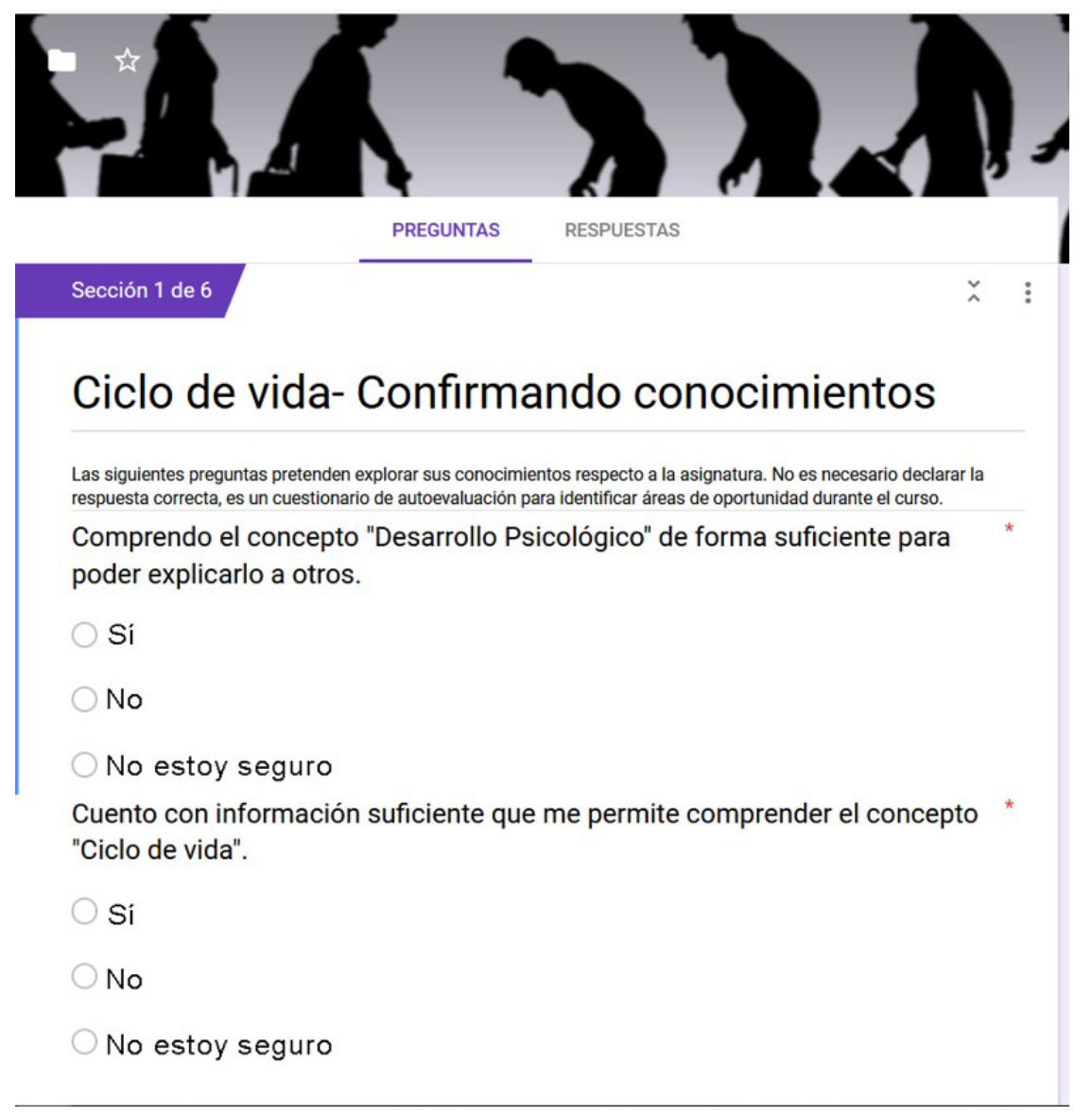

